\title{
Simultaneous Full-Field Multi-Experiment Identification
}

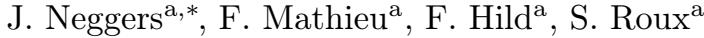 \\ ${ }^{a}$ Laboratoire de Mecanique et Technologie (LMT) \\ ENS Paris-Saclay / CNRS / Université Paris-Saclay \\ 61 avenue du Président Wilson, 94235 Cachan Cedex, France
}

\begin{abstract}
Identification of constitutive parameters relies mainly on their sensitivity to the measurands. In particular, the specific static and kinematic responses controlled by each parameter of interest has to be captured by full-field measurements. The development of modern constitutive models has led to many new and interesting sample geometries and loading histories, aiming at maximizing the sensitivity to their delicate material parameters, especially through their kinematics of interest. However, it is often impossible to design an experiment that activates all material parameters of interest, and thus multiple experiments are needed. This paper discusses a methodology for combining the data from such multiexperiments into a single identification process to calibrate a complete set of parameters at once. Many different ways of merging experimental data exist, leading to unbiased identifications of the parameters of interest. However, only one optimal procedure leads to minimal uncertainty, taking into account the noise of each acquisition source. The proposed identification method is a natural extension of inverse methods such as Finite Element Method Updating (FEMU) with appropriate weights or Integrated Digital Image Correlation (I-DIC). This procedure is illustrated by the identification of the planar parameters of the so-called Hill48 anisotropic yield surface together with an exponential isotropic hardening law of AA2219.
\end{abstract}

Keywords: Anisotropic plasticity, Digital image correlation, Full-Field

\footnotetext{
${ }^{*}$ Corresponding author

Email address: jan.neggers@ens-paris-saclay.fr (J. Neggers)
}

Preprint submitted to Mechanics of Materials

March 1, 2019 
measurements, Identification, Uncertainty

\section{Introduction}

Engineering applications are increasingly demanding fidelity of nonlinear constitutive models. The mechanics of materials community has been addressing this demand for many years. The field has significantly progressed in modeling

5 nonlinear mechanisms such as plasticity, viscosity, damage, fracture, delamination. Most of these modern constitutive models have in common that they require more material parameters often difficult to calibrate for a given material. This identification challenge hampers the usability of some of these models, thereby restricting their academic value and their industrial relevance [1, 2].

10

Fortunately, the experimental mechanics community is developing new methods to address these challenges. Currently, full-field measurement methods such as Digital Image Correlation (DIC [3, 4]) and the grid-method [5] have reached a data density level rich enough to identify multi-parameter nonlinear models. The increase in accuracy, efficiency and versatility of these full-field identification methods have greatly expanded the applicability of full-field identification methods $[6,7]$.

The most common identification method is referred to as Finite Element Model Updating (FEMU [6, 8-13]). In FEMU, the gap between the experiment and simulation of the same experiment is minimized by optimizing (i.e., updating) the unknown model parameters. Within this paper a similar method is applied, which is referred to as Integrated-DIC [14-20]. Integrated-DIC optimizes the gap between simulation and experiment directly on the captured images by integrating the identification step within the DIC algorithm. However, the differences between the two methods are not essential for the discussion in this paper. The interested reader is referred to Refs. $[18,21]$ for more details on this last point.

The goal of this paper is to propose a method that aggregates measurement results from difference sources into a single identification method while account- 
ing for the uncertainty of each measurement. The critical part is the proper weighting of each contribution. Some measurement sources consist of numerous data with poor quality (e.g., images) while others are limited in number but of higher quality (e.g., force measurements, strain gauges). The proposed method is defined such that, the weighting is not an arbitrary choice, but instead naturally follows from a Bayesian formulation given the number of measurements and their respective uncertainty of each source. The obtained weight is optimal, i.e., leading to the minimum uncertainty in the parameters for the case of Gaussian noise and insignificant model error.

Besides FEMU and Integrated-DIC there are other inverse identification methods (see e.g., $[6,7]$ for overview). The present paper focuses on these two

40 methods because both utilize the sensitivity fields in the same way. However, the concepts discussed herein may be equally valid for other identification methods such as the equilibrium gap method [22] or the virtual fields method [23, 24].

The proposed simultaneous multi-experiment identification method is presented using a proof of concept experiment. For the experiment, three uniaxial tensile tests were performed on Aluminum Alloy 2219 at the typical $0^{\circ}, 90^{\circ}$ and $45^{\circ}$ angles with respect to the rolling direction. Both classical identification and Integrated-DIC are performed and compared. The identified law is an elastoplastic material model with Ludwik (power law) isotropic hardening postulate [25], and the plane-stress Hill anisotropic yield surface [26]. It is well known that Hill's model is not optimal for describing the "anomalous" behavior often observed in aluminum alloys [2]. However, a perfect identification of the model is not the present goal. Validating that the multi-experiment implementation is able to calibrate an 8-parameter constitutive model using three simple uniaxial experiments is considered more important.

55 2. Methodology

For the application at hand, the experimental data will be the force measured by the load cell of the testing machine and the images of the sample 
surface recorded for each of the three experiments. It is important to propagate the measurement uncertainties along the entire identification chain. Therefore, the applied global DIC algorithm is briefly recalled, followed by a concise summary of single experiment identification using I-DIC to ultimately combine all ingredients into the multi-experiment identification method.

\subsection{Measurement Aggregation}

In the following, it will be shown that the weighting factor to be used is not a parameter to be chosen by the user but instead follows from the propagation of uncertainty. Consider $N$ observables, $x_{i}$ with $i=1, \ldots, N$, for which a model $\mathcal{G}$ can generate corresponding estimates from a set of $m$ parameters $\{p\}=$ $\left\{p_{1}, p_{2}, \ldots, p_{m}\right\}^{1}$

$$
x_{i}=\mathcal{G}_{i}(\{p\}) .
$$

Note that the observables $x_{i}$ can be of different types with different units (e.g., a set of measured forces and displacements). The measurement $\hat{x}_{i}$ of the observable $x_{i}$ is corrupted by Gaussian noise $\zeta_{i}$

$$
\hat{x}_{i}=x_{i}+\zeta_{i}
$$

The statistical distribution of $\zeta_{i}$ is the normal law $\mathcal{N}\left(0, \gamma_{i}^{2}\right)$ of zero mean and variance $\gamma_{i}^{2}$, thus the probability of the estimated observable to be equal to $x_{i}$ for each measurement $\hat{x}_{i}$ reads

$$
P_{i}=\frac{1}{(2 \pi)^{1 / 2} \gamma_{i}} \exp \left(-\frac{\left(\hat{x}_{i}-x_{i}\right)^{2}}{2 \gamma_{i}^{2}}\right)
$$

and hence for the entire set of $N$ measurements, assuming they are statistically independent, the probability reads

$$
P=\frac{1}{(2 \pi)^{N / 2} \prod_{i=1}^{N} \gamma_{i}} \exp \left(-\sum_{i=1}^{N} \frac{\left(\hat{x}_{i}-x_{i}\right)^{2}}{2 \gamma_{i}^{2}}\right) .
$$

\footnotetext{
${ }^{1}$ Throughout this article, the notations $\{\square\}$ and $[\square]$ are used to express column vectors and matrices, reserving the $\vec{\square}$ notation for vector quantities in $\mathbb{R}^{2}$ or $\mathbb{R}^{3}$.
} 
Inference of the most likely set of parameters that corresponds to a given set of measurements is equivalent to finding the maximum of Equation (4), or the minimum of the log-likelihood that is (up to irrelevant constants)

$$
\eta^{2}(\{p\})=\sum_{i=1}^{N} \frac{\left(\hat{x}_{i}-x_{i}\right)^{2}}{\gamma_{i}^{2}}
$$

The choice of the prefactor is such that the expectation of $\eta^{2}$ at convergence ${ }_{65}$ is $N$, assuming that the difference $\left(\hat{x}_{i}-x_{i}\right)$ is only due to noise. It is also noteworthy that the quadratic difference is not any arbitrary choice among many convex functions that are minimum at the origin, but the consequence of the Gaussian probability density function assumed for noise. At this point, typically nonlinear optimization methods are applied to find the optimal set of parameters that minimize Equation (5).

The most important conclusion from Equation (5) is that an identification using different aggregated measurement sets (in the presence of white noise) translates into minimizing the weighted sum of the squared differences. Taking the example of two sets of measurement data, with $N_{1}$ and $N_{2}$ measurements and variances $\gamma_{1}^{2}$ and $\gamma_{2}^{2}$ respectively, and labeled sequentially from 1 to $N=N_{1}+N_{2}$, the cost function is decomposed as

$$
\eta_{\text {total }}^{2}(\{p\})=\frac{1}{\gamma_{1}^{2}} \sum_{i=1}^{N_{1}}\left(\hat{x}_{i}-x_{i}(\{p\})\right)^{2}+\frac{1}{\gamma_{2}^{2}} \sum_{j=N_{1}+1}^{N}\left(\hat{x}_{j}-x_{j}(\{p\})\right)^{2} .
$$

This property endows the above cost function with a very convenient extensivity property that will be used farther down. This equation is equivalent to what is obtained from a variational approach or nonlinear least squares method [18, 19]. However, the important difference is that the weights that are required 75 to combine multiple cost functions follow from the derivation instead of being chosen arbitrarily. Choosing a different weight will still result in an unbiased identification, but it will overemphasize the importance of one measurement source as compared to the others, and hence lead to larger uncertainties. Let us also note that an intensive version can be defined, $\hat{\eta}^{2} \equiv \eta^{2} / N$, in which case so the total intensive cost function is the average of that of subgroups, weighted by 
the proportion of measurement number, a mathematically equivalent property convenient to assess model error when $\hat{\eta} \gg 1$, but requiring $N$ to be known for assembling different measurements.

The above case was written in the simple case of independent measurements. Correlated measurements can be treated in the very same framework and still result in a quadratic form to be minimized but now with a metric that is not Euclidean, but based on a kernel that is the inverse of the covariance matrix of the measurements [27]. If $C_{i j}$ denotes the covariance of the noise affecting measurements of $x_{i}$ and $x_{j}$, then the general expression of $\eta$ becomes

$$
\eta^{2}(\{p\})=\sum_{i, j=1}^{N}\left(\hat{x}_{i}-x_{i}\right) C_{i j}^{-1}\left(\hat{x}_{j}-x_{j}\right) .
$$

\subsection{Global DIC}

Digital image correlation aims at minimizing the difference in the observed intensity of a reference image $f$ and an image taken in the deformed state $g$ within some region of interest. The cost function that is minimized is defined as

$$
\eta_{\text {dic }}^{2}=\frac{1}{2 \gamma_{I}^{2}} \sum_{k=1}^{N_{k}}\left(f_{k}-\tilde{g}_{k}\right)^{2},
$$

85

where $N_{k}$ is the number of pixels considered in the region of interest, $f_{k}$ is the gray level of the $k^{\text {th }}$ pixel at the location $\vec{x}_{k}, \gamma_{I}^{2}$ the variance of the noise on each pixel, and $\tilde{g}_{k}$ the gray level at the location $\vec{x}_{k}+\vec{u}_{k}$ in the image $g$. A factor of $1 / 2$ has been included in the prefactor since noise affects both reference and deformed images, and hence with this factor the expectation of $\eta_{\text {dic }}^{2}$ is equal to

so the number of pixels. Note that $\tilde{g}$ typically requires gray level interpolation at non-integer pixel locations.

This DIC cost function is to be optimized with respect to the displacement field parameterized by a finite set of degrees of freedom. Since the proposed identification method already relies on finite elements, it is natural to adopt the very same finite element mesh for this purpose, for which the displacement field 
reads

$$
\vec{u}_{k}=\sum_{i=1}^{N_{a}} \vec{\varphi}_{k i} a_{i},
$$

where $a_{i}$ are the nodal degrees of freedom and $\vec{\varphi}_{k i}$ the corresponding finite element shape functions. In this paper, a single camera system is adopted resulting in 2D measurements. Ergo, the number of nodal degrees of freedom ${ }_{95} N_{a}$ is two times the number of mesh nodes.

Newton's method is then applied to iteratively minimize Equation (8) starting from an initial guess $\left\{a_{0}\right\}$. The solution at iteration $n+1$ is defined as

$$
\{a\}^{(n+1)}=\{a\}^{n}+\{\delta a\},
$$

where $\{\delta a\}$ is the iterative update. It is found by solving the linearized cost function

$$
\left[M^{\mathrm{dic}}\right]\{\delta a\}=\left\{b^{\mathrm{dic}}\right\},
$$

where

$$
\begin{aligned}
M_{i j}^{\text {dic }} & =\sum_{k=1}^{N_{k}}\left(\nabla f_{k} \cdot \vec{\varphi}_{k i}\right)\left(\vec{\varphi}_{k j} \cdot \nabla f_{k}\right), \\
b_{i}^{\text {dic }} & =\sum_{k=1}^{N_{k}} \nabla f_{k} \cdot \vec{\varphi}_{k i}\left(f_{k}-\tilde{g}_{k}\left(\{a\}^{n}\right)\right) .
\end{aligned}
$$

The above global DIC formulation is common and discussed in the literature (see e.g., Refs. [28, 29]).

Besides operating as the tangent matrix in the optimization algorithm, the Hessian $\left[M_{\mathrm{dic}}\right]$ serves a second purpose as its inverse represents the covariance matrix $\left[C^{\text {dic }}\right]$ if converged

$$
\left[C^{\mathrm{dic}}\right]=\left[M^{\mathrm{dic}}\right]^{-1},
$$

providing the means to relate gray level uncertainty to nodal displacement uncertainty while accounting for element size and pattern contrast variations. 


\subsection{Single-Experiment Identification}

The cost function for an Integrated-DIC routine is similar to the DIC cost function with the major change being that the unknowns now represent $N_{p}$ material parameters. A dimensionless version of the parameters is applied to allow for relative comparisons in sensitivity and uncertainty later on in the paper

$$
\{p\}=\left\{\frac{p_{1}}{p_{1}^{*}}, \frac{p_{2}}{p_{2}^{*}}, \ldots \frac{p_{N_{p}}}{p_{N_{p}}^{*}}\right\},
$$

where for the scaling values $p_{i}^{*}$ the initial parameters are used (i.e., $\left.\left\{p_{0}\right\}\right)$. The Integrated-DIC cost function then becomes

$$
\eta_{I}^{2}(\{p\})=\frac{1}{2 \gamma_{I}^{2}} \sum_{t=1}^{N_{t}} \sum_{k=1}^{N_{k}}\left(f_{k}-\tilde{g}_{k t}(\{p\})\right)^{2},
$$

where $N_{t}$ is the number of time steps considered for identification and $\gamma_{I}^{2}$ the estimated gray level variance for each acquisition. The additional summation over time reflects the fact that the constitutive parameters are equally valid for all time steps and according to Section 2.1, the different log-likelihood functionals are to be simply summed. Hence, the gray level $\tilde{g}_{k t}$ is taken at the location $\vec{x}_{k}+\vec{u}_{k t}$ of the image acquired at time step $t$. The displacement field $\vec{u}_{k t}(\{p\})$ is provided by an FE simulation using the current estimate of the parameters.

Similarly additional cost functions can be defined for other observables that will be used for identification purposes. In the test case discussed hereafter, besides images, the reaction force at one of the grips is also utilized

$$
\eta_{F}^{2}(\{p\})=\frac{1}{\gamma_{F}^{2}} \sum_{t=1}^{N_{t}}\left(F_{t}^{\exp }-F_{t}(\{p\})\right)^{2},
$$

where $F_{t}^{\exp }$ and $F_{t}$ are respectively the measured and simulated resultant force at the sample boundary at time step $t$. Similarly as with Equation (16), the cost 110 function is normalized using the estimated variance for each measurement $\gamma_{F}^{2}$.

Exactly as in Equation (6) in Section 2.1, the cost functions for each observable is combined by summation in the normalized forms as expressed in Equations (16) and (17),

$$
\eta_{e}^{2}=\eta_{I}^{2}+\eta_{F}^{2}
$$


The solution to this inverse problem is obtained by iteratively minimizing this cost function using Newton's method starting with an initial guess for the parameters $\left\{p_{0}\right\}$

$$
\{p\}^{(n+1)}=\{p\}^{n}+\{\delta p\},
$$

where the incremental update to the parameters $\{\delta p\}$ is found by solving

$$
\left(\left[M_{I}\right]+\left[M_{F}\right]\right)\{\delta p\}=\left\{b_{I}\right\}+\left\{b_{F}\right\},
$$

with

$$
\begin{aligned}
& {\left[M_{I}\right]=\frac{1}{2 \gamma_{I}^{2}}\left[S_{I}\right]^{\top}\left[M^{\mathrm{dic}}\right]\left[S_{I}\right],} \\
& \left\{b_{I}\right\}=\frac{1}{2 \gamma_{I}^{2}}\left[S_{I}\right]^{\top}\left\{b^{\mathrm{dic}}\right\},
\end{aligned}
$$

and

$$
\begin{aligned}
& {\left[M_{F}\right]=\frac{1}{\gamma_{F}^{2}}\left[S_{F}\right]^{\top}\left[S_{I}\right],} \\
& \left\{b_{F}\right\}=\frac{1}{\gamma_{F}^{2}}\left[S_{F}\right]^{\top}\left\{F^{\exp }-F\right\},
\end{aligned}
$$

where $\left[S_{I}\right]$ and $\left[S_{F}\right]$ represent the displacement and the force sensitivities. They are rectangular matrices projecting from the node-time space to the parameter space and follow from the derivation using Newton's method

$$
\begin{gathered}
\left\{S_{I i}\right\}=\frac{\partial\{a\}}{\partial p_{i}}, \\
\left\{S_{F i}\right\}=\frac{\partial\{F\}}{\partial p_{i}} .
\end{gathered}
$$

In some special cases, analytical derivatives are obtainable (see e.g., Refs. [3033]). However, for most constitutive models, approximative derivatives have to be evaluated, as for example via finite differences. In the above equations, the sensitivity matrices are computed by performing one simulation using the current estimate of the parameters to obtain the reference nodal values $\{a\}$ and $\{F\}$ and a second one per each unknown $p_{i}$ where the parameter is increased by $1 \%$. 
Another interpretation of Equation (21) is that the displacement field (instead of the images) is considered the measurand and the $\left[C^{\text {dic }}\right]$ matrix is applied as the metric with which to transport covariance from a previous measurement (DIC) to the identification method. This observation highlights that the build-up of covariance is transparent to the identification method if it is consistently transported along the measurement chain (see also Equation 7). In other words, performing FEMU (with the displacement field as measurand), 125 results in exactly the same cost function definition as I-DIC (using the image as measurand), provided the covariance accumulated during the DIC phase is taken into account (see also Ref. [18]).

\subsection{Multi-experiment identification}

The premise that drives multi-experiment identification is that the constitutive parameters are valid for a range of experiments of the studied material. Consequently, different experiments may be performed to activate different material parameters. While some experiments may be relevant to trigger sensitivity for specific parameters, other parameters will be active in all experiments. The proposed method of measurement aggregation (see Section 2.1) and its adoption in the previous section to aggregate multiple data sources into a single experiment is extended to aggregate data from multiple experiments

$$
\eta^{2}=\sum_{e=1}^{N_{e}} \eta_{e}^{2}
$$

where the total cost function $\eta^{2}$ is simply the sum of the individual cost functions

for each experiment, as defined in the previous section, $N_{e}$ being the total number of experiments.

The chosen solution strategy remains unchanged from Newton's method as defined in Equation (19), where the update to the degrees of freedom obtained by solving the linearized system of equations reads

$$
[M]\{\delta p\}=\{b\}
$$


where the Hessian $[M]$ and the right hand member $\{b\}$ are the sums of the "per experiment" respective contributions

$$
[M]=\sum_{e=1}^{N_{e}}\left(\left[M_{I e}\right]+\left[M_{F e}\right]\right), \quad\{b\}=\sum_{e=1}^{N_{e}}\left(\left\{b_{I e}\right\}+\left\{b_{F e}\right\}\right),
$$

thereby resulting in one global Hessian and right hand member seamlessly combining the data from multiple experiments. Iteratively solving this system of equations will result in finding a single set of parameters that optimally minimizes the discrepancy between multiple experimental sets of data and multiple simulations as gauged by their uncertainty.

\subsection{Identification framework}

The previous sections have introduced the general identification procedure. However, details still remain to be discussed for the method to become practical.

Boundary Conditions. The previously discussed approach relies on simulating the experiment using FE models. The quality of the parameter identification relies on the simulations to mimic the experiments as closely as possible and thus they need to be driven with accurate boundary conditions. The chosen method is to use a regular (i.e., non-integrated) DIC routine to measure the displacements near the edges of the field of view and use these displacements as boundary conditions for the simulation. These displacements are measured by DIC and thus are affected by noise creating an alternative way for measurement noise to influence the identification. The influence of this noise is an ongoing research subject and is not considered in the analyses and results [34]. However, effort is made to minimize this influence, which will be discussed in Section 2.9. On the other hand, the method of using the DIC measured boundary conditions has a few advantages, namely, it limits the simulation domain to the region of interest, it provides time synchronization, it naturally deals with any sample misalignment and rigid body motion [35].

Conditioning. Although the proposed method condenses a large amount of data to a relatively smaller system of equations to be solved (Equation (28)), the 
system can still be poorly conditioned. Reasons for ill-conditioning can be, for example, insufficient sensitivity to a given parameter (or linear combinations thereof). To limit the impact of these issues, Tikhonov regularization is applied [36]

$$
([M]+\alpha[I])\{\delta p\}=\{b\}+\alpha\left(\left\{p_{\text {ref }}\right\}-\{p\}\right),
$$

155 where $[I]$ is the identity matrix, $\alpha$ the regularization strength set to $\alpha=10^{-5} \lambda$, where $\lambda$ is the largest eigenvalue of $[M]$ and $\left\{p_{\text {ref }}\right\}$ is a set of parameters obtained from external sources such as other experiments of the literature (here chosen as $\left\{p_{0}\right\}$ the initial guess of the minimization procedure). The regularization strength is set sufficiently low such that parameters with sufficient sensitivity will be calibrated by the multi-experiment identification while suppressing spurious behavior of the ill-conditioned parameters. Note that when $\alpha=0$ the original system is obtained.

Secant method. For the proposed method, most of the cost is in the finite element simulations. Consequently, updating the sensitivity matrices (Equation (25) and 26) is costly, since they require $N_{p}+1$ simulations. Therefore, within this paper the sensitivity matrices are not updated for every iteration. This effectively modifies Newton's scheme to a secant method. The update in the sensitivity matrices is only performed when the cumulative update in the parameters since the last sensitivity update is larger than $5 \%$.

Algorithm Summary. The implemented framework consists of the following steps:

1. Measure the boundary conditions using DIC

2. Choose the initial parameter set $\left\{p_{0}\right\}$

3. Compute the reference displacement field and force for each experiment

4. If large changes in parameters, compute the sensitivity matrices for each experiment (Equations (25) and (26))

5. Aggregate the data from all experiments (Equation (29)) 
6. Solve for the correction in the parameters (Equation (28))

7. Update the parameters (Equation (19))

8. If not converged return to step 3

Most of the computational cost lies in steps 3 and 4 since they involve a number of often heavy finite element simulations. However, these simulations can be performed concurrently while each of them can be parallelized. This can greatly reduce computation time if the computational resources are available. Typically step 5 can be performed within a few minutes for a moderate data set on a normal desktop computer and thus its cost will only be significant for cases with comparable finite element simulation costs.

\subsection{Experiment}

The three typical tensile experiments treated in this paper are those that are commonly performed for identifying planar anisotropic yielding [37, 38]. The samples were cut such that their respective loading axes aligned with three important rolling direction, namely the Longitudinal (L), Transverse (T) and Diagonal (D) directions. The longitudinal direction was aligned with the rolling direction of the sheet at $0^{\circ}$. The transverse sample was cut at $90^{\circ}$ while the diagonal sample was cut at $45^{\circ}$ with respect to the rolling direction, as shown in Figure 1. These sample directions will be denoted as L, T and D henceforth.

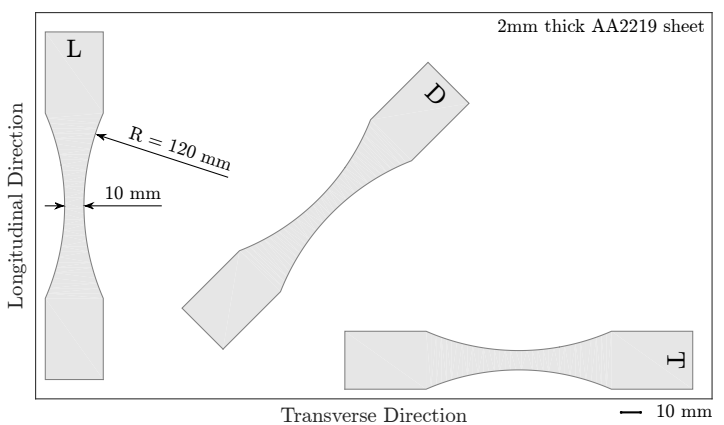

(a) Samples

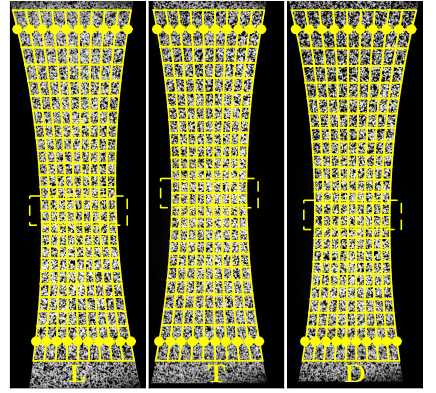

(b) Mesh

Figure 1: (a) Orientation of the samples as cut from the aluminum alloy sheet. (b) Picture of the three samples with their speckle pattern and conforming mesh. 
Each sample was $2 \mathrm{~mm}$ thick and $180 \mathrm{~mm}$ long, the width at the narrowest section was $10 \mathrm{~mm}$, with the dog-bone radius of each side of the sample equal to $120 \mathrm{~mm}$, see Figure 1. The samples ware loaded in a servohydraulic tension/compression testing machine equipped with a $50 \mathrm{kN}$ load-cell. The top grip of the tensile machine was stationary while the bottom grip was driven at constant velocity of $0.01 \mathrm{~mm} / \mathrm{s}$ to specific load levels (i.e., [1.8, 6.0, 8.5, 9.1, 9.3, $9.5,9.6,9.65[\mathrm{kN})$. When a load level was reached the sample was unloaded to $0.1 \mathrm{kN}$ upon which the next loading cycle starts.

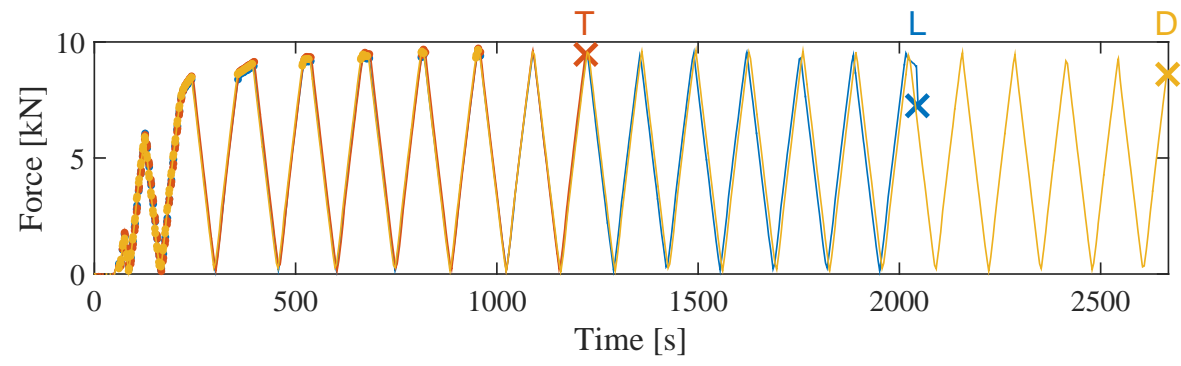

Figure 2: Force response over time for all three experiments. This figure shows all experimental cycles the three samples, indicating where each sample ultimately failed (marked $\mathbf{X}$ ) and the images that were considered herein (marked $\bullet$ ). Note that the first two cycles wee used with unloading, while upon plasticity only the parts where the force was monotonically increasing are used up to the $8^{\text {th }}$ cycle.

Only the first 8 cycles are considered as the experimental dataset in this paper. Moreover, for the "plastic" cycles, only the images where the force was monotonically increasing are kept in the dataset (Figure 2). The later cycles were discarded because the applied constitutive model (to be introduced below) was not rich enough to allow for parameter calibration after the onset of necking. Each experiment was started holding the load at $0 \mathrm{~N}$ while capturing 20 images. These images are only used to estimate the measurement uncertainty. The identification dataset is based on $[114,110,114]$ images per experiment respectively, 1 reference and $[113,109,113]$ deformed increments containing two elastic load-unload cycles and the monotonic loading part up to $9.65 \mathrm{kN}$ of 215 load. 
The images were captured with a single Manta G-223 camera equipped with a telecentric lens of magnification $\times 0.125$, and captured one side of the specklepainted sample twice at $5 \mathrm{~s}$ intervals, once at $1 \mathrm{~s}$ and once at $5 \mathrm{~s}$ in each interval. The recorded images are of definition $1120 \times 2160 \mathrm{px}$, where each pixel captures plotting style is adopted throughout the paper that merits a few words of explanation (see e.g., Figure 3). The 3D data volume is represented by two 2D cross-sections, the first is in the time- $y$ plane taken at $x=[200,145,170] \mathrm{px}$ respectively for the $\mathrm{L}-, \mathrm{T}$ - and $\mathrm{D}$-samples, the second is in the $x-y$ plane taken in the figures with dashed lines in the other cross-section. Furthermore, various vector components of the fields are plotted as rows of sub-figures. Single point data (e.g., force) are plotted when relevant using the same time axes below the final vector/tensor component time- $y$ figure. In this visualization style all 245 data considering a single experiment can be compactly summarized in a vertical arrangement, allowing the data of the three experiments $(\mathrm{L}, \mathrm{T}$ and $\mathrm{D})$ to be 
plotted side by side for easy comparison.
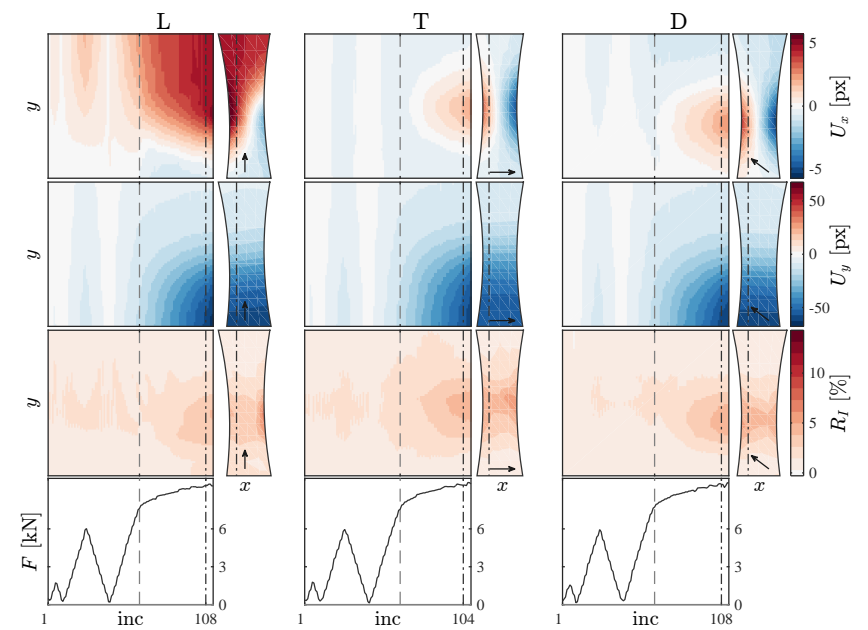

Figure 3: Displacement field (in space and time) as measured by non-integrated DIC for all three experiments (rows 1 and 2), and the respective image residuals (row 3) and the measured force at the load-cell (row 4).

Figure 3 summarizes the full-field space-time displacement data as obtained by DIC for all three experiments. In the experiments, the top grip of the tensile of view is smaller than the clamped sample length (i.e., $\sim 48 \mathrm{~mm}$ ) both top and bottom mesh boundaries are moving vertically. The L-sample was mounted with a visible misalignment that resulted in a significant horizontal motion of the bottom mesh boundary due to the lateral freedom of the bottom grip. Due to the limited number of samples, the experiment was not discarded but the data were used as-are. Fortunately, the adopted full-field identification method is naturally tolerant to these real-word experimental issues.

DIC is also applied to the first 20 images, captured without load on the sample. The root-mean-square (RMS) of the image residuals, standard deviation of the nodal displacements and the standard deviation of the measured force over these 19 time steps are used as estimates of the respective measurement 
uncertainties

$$
\begin{aligned}
\gamma_{I} & \approx 1911 \mathrm{GV} \approx 2.9 \% \\
\gamma_{U} & \approx 0.017 \mathrm{px} \approx 0.44 \mu \mathrm{m} \\
\gamma_{F} & \approx 5 \mathrm{~N}
\end{aligned}
$$

where the gray level uncertainty is computed using

$$
\gamma_{I}=\frac{1}{6} \sqrt{6} G\left\langle\gamma_{U} L\right\rangle
$$

where $G$ is the mean field average of the image gradient and $L$ the element length which is approximated as the square root of the average element area [29, 39]. uncertainty being approximately $2.9 \%$ of the dynamic range. This method of obtaining the gray level uncertainty is used to be consistent with a previous study that used the same images [20]. In the latter reference, this estimate was introduced as an effective gray level uncertainty that also accounts for gray level variations from other sources such as cross-pixel correlation, speckle degradation and sub-pixel interpolation errors. The impact of this choice is currently under research but is estimated to be small.

\subsection{Constitutive Model}

The constitutive model chosen to be identified using the three experiments is an elastoplastic law with isotropic exponential hardening and an anisotropic yield surface. The chosen hardening law is that proposed by Ludwik [25]. In a $1 \mathrm{D}$ setting, under increasing plastic strain $\varepsilon_{p}$ the stress $(\sigma)$ strain $(\varepsilon)$ relationship reads

$$
\begin{array}{ll}
\sigma=E \varepsilon, & \sigma \leq \sigma_{0}, \\
\sigma=\sigma_{0}+h \varepsilon_{p}^{m}, & \sigma>\sigma_{0},
\end{array}
$$

where $E$ is the Young's modulus, $\sigma_{0}$ the yield stress, $h$ the hardening modulus, and $m$ the hardening power. Together with Poisson's ratio $\nu$, this model results in five unknown material parameters. The anisotropic yield surface is described 
with Hill's model [26], which is readily available in Abaqus [40]. The anisotropic plasticity criterion is defined as

$$
\begin{aligned}
H_{1}\left(\sigma_{22}-\sigma_{33}\right)^{2}+H_{2}\left(\sigma_{33}-\sigma_{11}\right)^{2}+ & H_{3}\left(\sigma_{11}-\sigma_{22}\right)^{2} \\
& +2 H_{4} \sigma_{23}^{2}+2 H_{5} \sigma_{31}^{2}+2 H_{6} \sigma_{12}^{2}=1,
\end{aligned}
$$

with

$$
\begin{aligned}
H_{1} & =\frac{1}{2}\left(\frac{1}{R_{22}^{2}}+\frac{1}{R_{33}^{2}}-\frac{1}{R_{11}^{2}}\right), & H_{4} & =\frac{3}{2} \frac{1}{R_{23}^{2}}, \\
H_{2} & =\frac{1}{2}\left(\frac{1}{R_{33}^{2}}+\frac{1}{R_{11}^{2}}-\frac{1}{R_{22}^{2}}\right), & H_{5} & =\frac{3}{2} \frac{1}{R_{13}^{2}}, \\
H_{3} & =\frac{1}{2}\left(\frac{1}{R_{11}^{2}}+\frac{1}{R_{22}^{2}}-\frac{1}{R_{33}^{2}}\right), & H_{6} & =\frac{3}{2} \frac{1}{R_{12}^{2}},
\end{aligned}
$$

where $R_{11}, R_{22}, R_{33}, R_{12}, R_{13}$ and $R_{23}$ are yield stress ratios. For a near planefully describe the yield surface [2]. Therefore, the parameters $R_{22}, R_{33}, R_{12}$ and $\sigma_{0}$ are used adding 3 parameters to the identification routine. A large transformation framework is used in the FE simulations.

\subsection{Classical Identification Methods}

275

A more classical identification approach is first applied before using the proposed full-field multi-experiment identification. This analysis serves two purposes, $(i)$ provide a set of parameters $\left\{p_{0}\right\}$ to initialize the identification, (ii) provide a reference to compare the identified parameters with. This classical identification is not considered as a golden standard, yet for the test case at hand, both methods are within their application range and should provide comparable results.

The non-integrated DIC displacement measurements are used to compute the logarithmic strain fields that are shown in Figure 4. A virtual strain gauge is applied by averaging the strains in the center region of the samples indicated by the dashed box in Figure 1(b). 

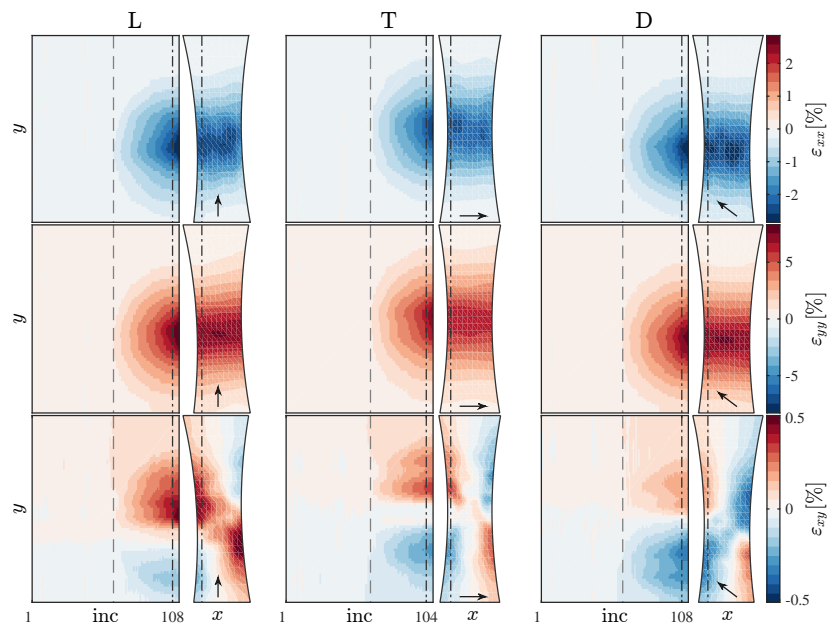

Figure 4: Strain fields (in space and time) as measured by non-integrated DIC for all three experiments.

The stress in the tensile direction of the sample (i.e., $y$-direction) is estimated using the measured force $F$, the cross-sectional area in the reference state $A_{0}$ and the stretch in the same direction

$$
\sigma_{i y y} \approx \frac{F_{i} \exp \left(\varepsilon_{i y y}\right)}{A_{0}}, \quad i=[L, T, D],
$$

Equation (34) is fitted to the L-data using Newton's method to obtain the parameters $E, \sigma_{0}, h$ and $m$. The ratio between the $x x$ - and $y y$-components of strain for the sample experiment are used to identify $\nu$. The result of the stress-strain curve obtained by the fit is shown with a dashed line in Figure 5(a). 

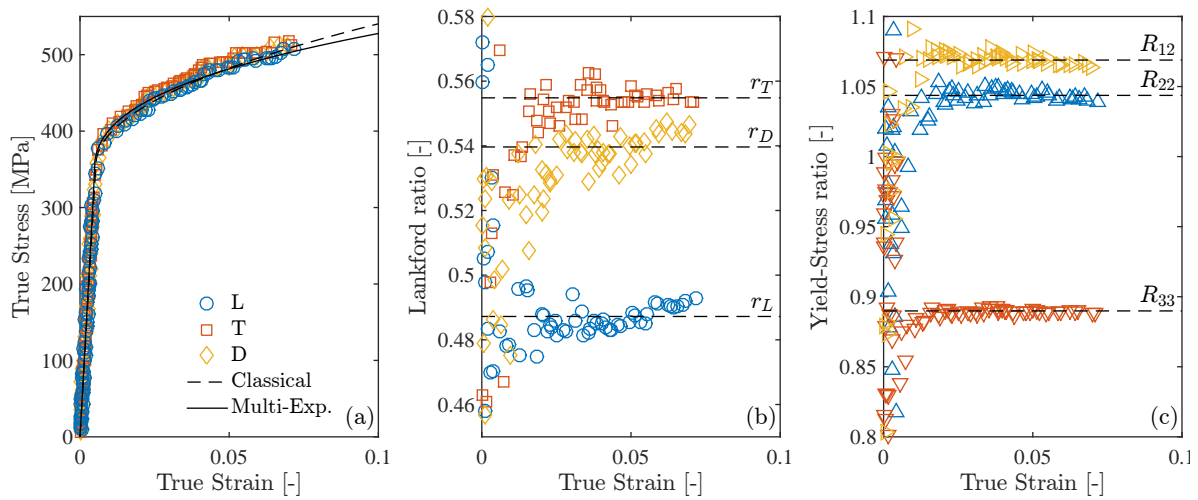

Figure 5: Plots of (left) stress-strain data for all three experiments, (center) Lankford ratio vs. strain and (right) Hill's yield stress ratio vs. strain

From each experiment Lankford's ratios are extracted using the plastic strains $\varepsilon_{p}$ from the two directions in the cross-sectional plane (i.e., the $x x$ - and $z z$ directions)

$$
r_{i}=\frac{\varepsilon_{p_{i x x}}}{\varepsilon_{p_{i z z}}}, \quad \quad i=[L, T, D],
$$

where the plastic strains in those directions are estimated using the total strain the $x x$ - and $y y$-directions and the parameters identified in the previous section

$$
\begin{aligned}
\varepsilon_{p_{x x}} & =\varepsilon_{x x}+\nu \frac{F}{A E}, \\
\varepsilon_{p_{y y}} & =\varepsilon_{y y}-\frac{F}{A E}, \\
\varepsilon_{p_{z z}} & =\log \left(\frac{1}{\exp \left(\varepsilon_{p_{x x}}+\varepsilon_{p_{y y}}\right)}\right) .
\end{aligned}
$$

The Lankford ratios for each time increment are shown in Figure 5(b).

Last, the three yield stress ratios are obtained from the three Lankford ratios

$$
\begin{aligned}
& R_{22}=\frac{\sigma_{22}}{\sigma_{0}}=\sqrt{\frac{r_{T}\left(r_{L}+1\right)}{r_{L}\left(r_{T}+1\right)}} \\
& R_{33}=\frac{\sigma_{33}}{\sigma_{0}}=\sqrt{\frac{r_{T}\left(r_{L}+1\right)}{r_{L}+r_{T}}} \\
& R_{12}=\frac{\sigma_{12}}{\tau_{0}}=\sqrt{\frac{3 r_{T}\left(r_{L}+1\right)}{\left(2 r_{D}+1\right)\left(r_{L}+r_{T}\right)}}
\end{aligned}
$$


where 1, 2 and 3 indicate directions corresponding to the material orientation, 1 and 2 are in the plane of the metal sheet while 3 is perpendicular to the plane of the sheet. The yield stress ratios for each time increment are shown in Figure 5(c). The values above $3 \%$ strain are averaged to identify the yield stress ratios.

\subsection{FE Simulations}

The simultaneous multi-experiment identification algorithm detailed in the previous section is implemented within the Correli 3.0 framework, which is in continuous development at LMT [41]. The framework contains the tools to perform non-integrated DIC, manage the launch and data retrieval of all the FE simulations. For this paper Abaqus ${ }^{2}$ is used to perform the FE simulations. In general, Integrated-DIC treats the simulation software as a "black box" where each simulation is started with a different set of parameters and the only exploited output data are the displacement fields and nodal forces for each time step.

The FE meshes used for the simulations are based on those shown in Figure 1(b) with two modifications. The first is the removal of one row of elements from each side of the sample (see Figure 6). This is done so that the applied boundary conditions are those measured by DIC on internal nodes, which are less sensitive to acquisition noise compared to boundary nodes [29]. To further reduce the impact of measurement noise the measured displacements are filtered using $4^{\text {th }}$ order polynomials [42]. The second modification to each mesh is that it is extruded in the $z$-direction to create a 3D model (see Figure 6). Even though the applied measurement is $2 \mathrm{D}$, there is no limitation on performing the simulations in 3D. It was shown that it is desirable when dealing with elastoplastic laws [35].

\footnotetext{
${ }^{2}$ Abaqus Standard: Dassault Systèmes Simulia [40]
} 


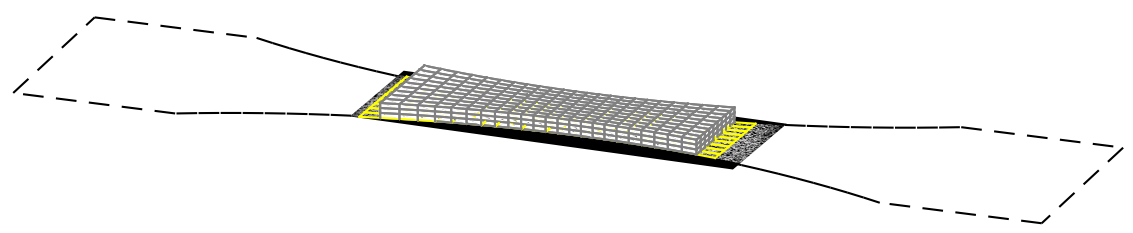

Figure 6: Complete sample shape (dashed line) with the image positioned on top with the 2D DIC mesh (yellow) and the 3D FE mesh.

\section{Identification Results}

The main objective of identification is the set of parameters. However, the proposed method also allows for the analysis of the obtained result providing understanding about the sensitivity and uncertainty for each parameter. The analysis is mainly based on the sensitivity matrices (Equations (25) and (26)), the Hessians (Equations (21), (23) and (29)) and the full-field residuals.

\subsection{Sensitivity Matrices}

Figure 7 shows the sensitivity fields for the five isotropic parameters for the L-experiment. Similar figures can be created for the other two experiments. However, these will not show significantly different results. The figure is composed of five columns of five sub-figures each. Each column corresponds to one parameter. The displacement component of the sensitivity matrix for one parameter has the same data structure as a displacement field, which is in this case 3-dimensional with two dimensions in space and one in time. Consequently, the same representation can be used as shown in Figure 3. The full field data in the displayed figures (first and second rows) are scaled per parameter with the amplitude that is given above the column $\bar{p}_{i}$. This normalization is mainly performed to aid visualization since it brings all the drawn fields within the same $[-1,1]$ range allowing a single color bar. Since the parameters $\{p\}$ are dimensionless, the units of the sensitivity matrices are equal to the respective measured quantities, namely, px for the displacements and $\mathrm{N}$ for the force sensitivity. 

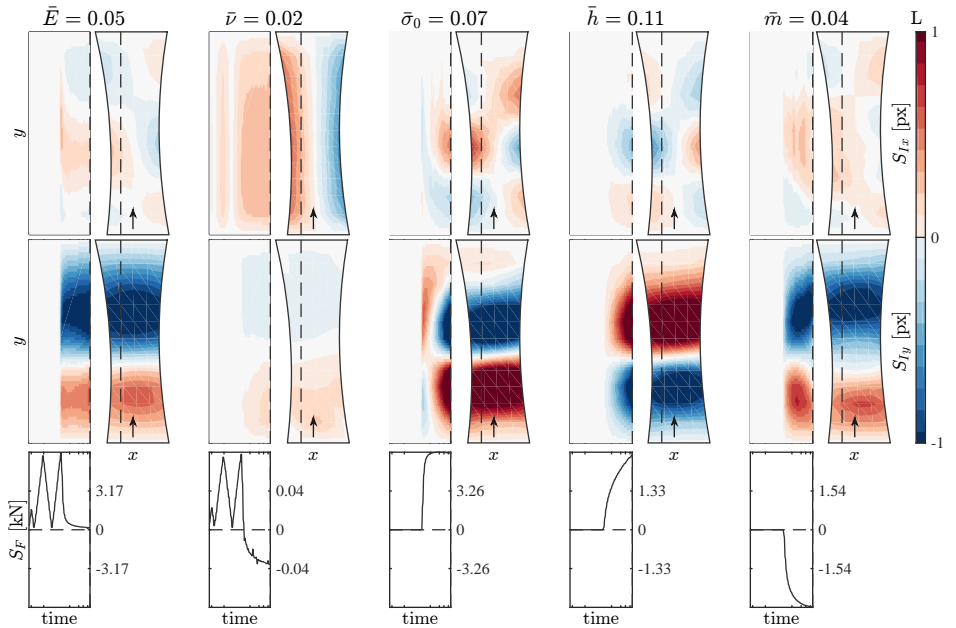

Figure 7: Sensitivity fields in terms of the $x$ - and $y$-displacement and force sensitivity for the five isotropic parameters for the L-sample each scaled with the amplitude $\bar{p}$ given on top.

Figure 7 quantitatively shows where in space and time each parameter is sensitive. For instance, it illustrates that the second parameter $\nu$ has the weakest sensitivity, indicated by its normalization factor $\bar{\nu}=0.02 \mathrm{px}$ and its force amplitude being approximately $0.05 \mathrm{~N}$. Both are within the expected measurable range and thus this parameter should be tunable. However, it will be the most prone to measurement noise. It should be noted that it is possible to compute these figures before performing the experiment. Consequently, they are a useful tool for optimizing the experiment to enhance the sensitivity of certain parameters [34]. For example, as expected, most of the sensitivity for the plastic parameters comes from the end of the experiment.

Figure 8 shows the sensitivity fields for the first anisotropic parameter (i.e., $R_{22}$ ) for each of the performed tests (i.e., L, T, and D). As expected, the contribution of each experiment to the sensitivity is distinct. Interestingly, the $\mathrm{L}$ and $\mathrm{D}$ experiments show the strongest displacement sensitivity while the $\mathrm{T}$ experiment shows the strongest force sensitivity. The difference in sensitivity in the force part is much more significant showing a 50 times increase in sensitivity for the $\mathrm{T}$ experiment compared to the $\mathrm{L}$ experiment. 

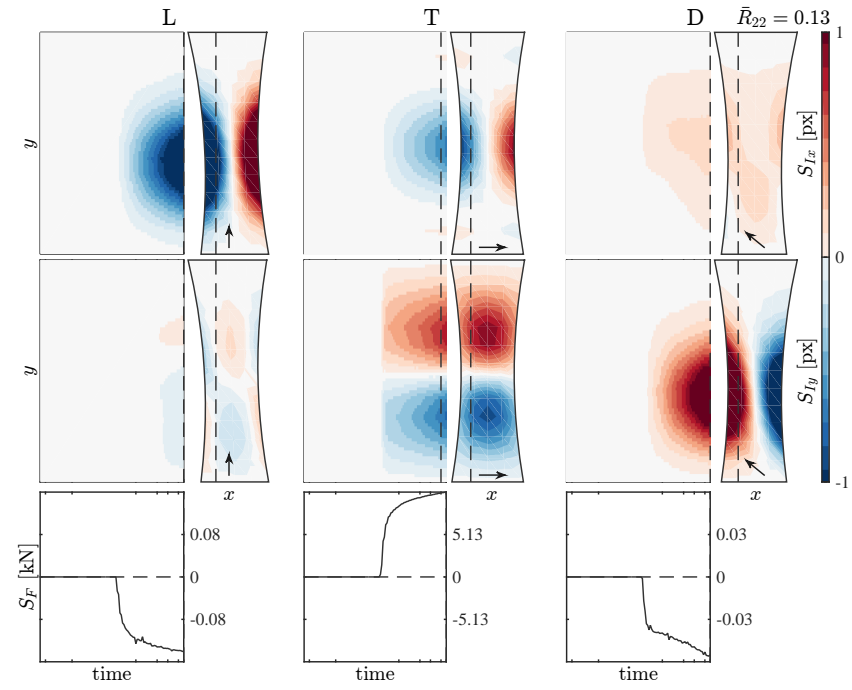

Figure 8: Sensitivity fields for the anisotropic stress ratio $R_{22}$ for each of the three tensile directions (L, T and D).

Figure 9 shows similar results for the other two anisotropic stress ratios $R_{33}$ and $R_{12}$. The $R_{33}$ stress ratio shows equivalent kinematic sensitivity fields for all three experiments while the static sensitivity is approximately 10 times stronger for the $\mathrm{D}$-sample. As expected, the shear stress ratio $R_{12}$ requires the $45^{\circ}$ experiment to activate significant sensitivity. This sensitivity field analyses show that all three anisotropic parameters are significantly activated in one or more experiments both from kinematic and static points of view. Sensitivity for the $R_{22}$ parameter depends mostly on the T-sample while $R_{33}$ and $R_{12}$ mostly on the D-sample. Interestingly, it seems that the L-sample is not significantly carrying sensitivity not already present in the other two experiments. The data from the L-sample to the identification will however increase the sensitivity for these three and the five isotropic parameters. Since uncertainty is inversely related to sensitivity it is advised to use all three experiments. However, if some uncertainty can be tolerated, the experimental load can be reduced by omitting the L-experiment. 

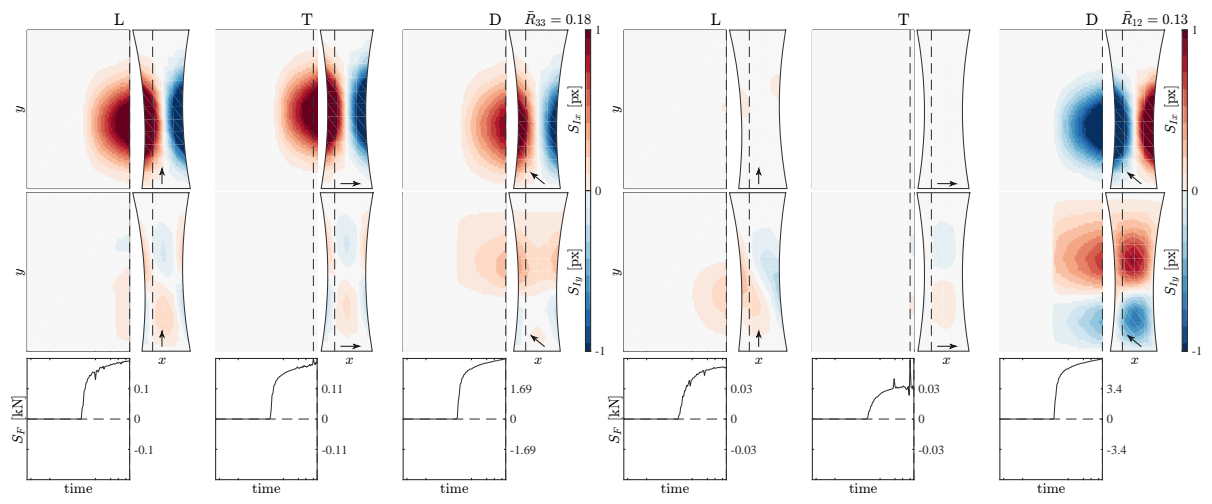

(a) $R_{33}$

(b) $R_{12}$

Figure 9: Sensitivity fields for the anisotropic stress ratios $R_{33}$ (a) and $R_{12}$ (b) for each of the three tensile direction ( $\mathrm{L}, \mathrm{T}$ and $\mathrm{D})$.

370

\subsection{Uncertainty quantification}

The full-field sensitivity analysis is effective for analyzing where in space and time each parameter is active. However, the final system of equations that is solved during the iterative process is a condensed version of the sensitivity fields (see Equation (29)). The Hessian is the sum of many sub-Hessian matrices, namely, one displacement and one force per experiment. Each experiment generates two sub-Hessians, only the two for the L-experiment are visually represented in Figure 10. This figure shows that the image (or kinematic) part of the measurement carries a significant portion of the sensitivity. It also shows that some groups of parameters are dependent on each other. This is visible from a block diagonal aspect of the Hessian. However, to further interpret the structure of this Hessian, one should consider the eigenvectors and eigenvalues of $[\mathrm{M}]$, as the presence of off-diagonal terms means that the actual uncertainty is more intricate. This is considered out of the scope of this paper. 


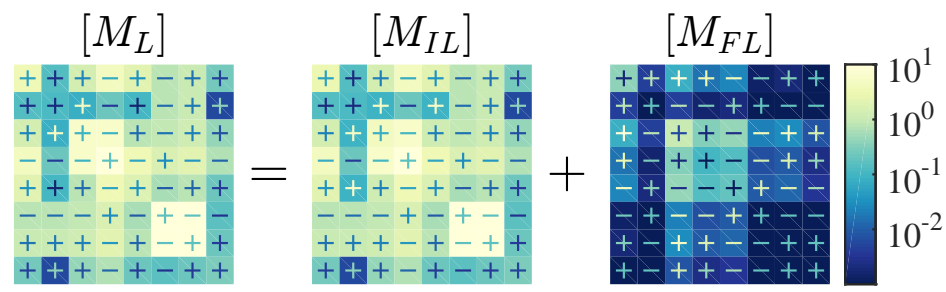

Figure 10: Hessian matrix for the L-sample $\left[M_{L}\right]$, which is the sum of its kinematic part $\left[M_{I L}\right]$ and static part $\left[M_{F L}\right]$. The matrix rows and columns correspond to the constitutive parameters in order $E, \nu, \sigma_{0}, h, m, R_{22}, R_{33}, R_{12}$.

Figure 11(a) shows the aggregation of the per experiment Hessian matrices into a single Hessian. Note how the sensitivity accumulates from the three experiments. The parameter sensitivity of the three experiments is perhaps more clearly illustrated in the covariance matrix, which is the inverse of sensitivity, namely, uncertainty (see Figure 11(b)). For Gauss-Newton algorithms, the covariance matrix can be approximated by the inverse of the Hessian in the converged state [43]. Equivalently as the Hessian matrix expresses sensitivity, so is uncertainty expressed by the covariance matrix. Combining the three experiments into one identification results in a significantly lower variance and covariance for the anisotropic parameters. To show the correlation between parameters, the covariance matrix is normalized to obtain the correlation matrix (no index summation)

$$
Q_{i j}=\frac{M_{i j}^{-1}}{\sqrt{M_{i i}^{-1} M_{j j}^{-1}}},
$$

which is shown in Figure 11(c). The two elastic parameters $E$ and $\nu$ are relatively uncorrelated, the three plastic parameters $\sigma_{0}, h$ and $m$ are strongly correlated as are the three anisotropy parameters. In general, correlation of parameters does not lead to identification issues as long as there are no double eigenvalues or near zero eigenvalues in the set of calibrated parameters. However, correlation does complicate the discussion revolving about uncertainty, since the uncertainty of one parameter is influenced by other parameters. 
(a)

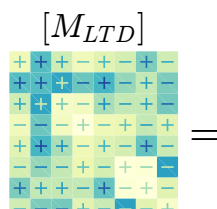

$\left[M_{L}\right]$

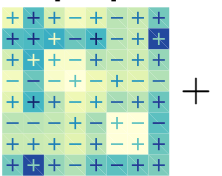

$\left[M_{T}\right]$

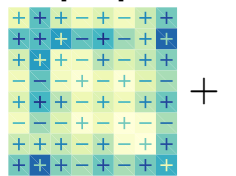

$\left[M_{D}\right]$

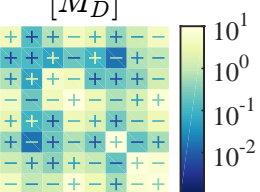

[C $\left.C_{L T D}\right]$

$\left[C_{L}\right]$

$\left[C_{T}\right]$

(b)
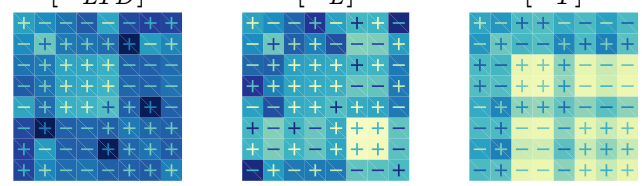

$\left[C_{D}\right]$

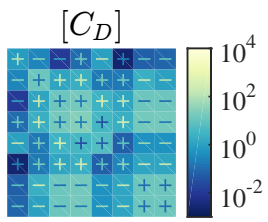

$\left[Q_{L T D}\right]$

(c)

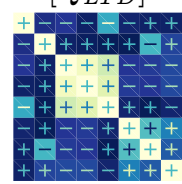

$\left[Q_{L}\right]$

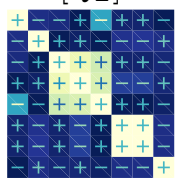

$\left[Q_{T}\right]$

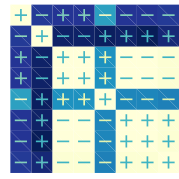

$\left[Q_{D}\right]$

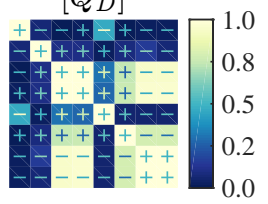

Figure 11: (a) Total Hessian $\left[M_{L T D}\right]$, which is the sum of the per-experiment Hessians $\left(\left[M_{L}\right]\right.$, $\left[M_{T}\right]$ and $\left.\left[M_{D}\right]\right)$. (b) Inverse of each matrix shown above, which are approximations of the covariance matrices. (c) Correlation matrices, which are obtained by normalizing covariance matrices. The colors represent the absolute values of the components, while the signs are indicated with "+" and "-". The constitutive parameter order is $E, \nu, \sigma_{0}, h, m, R_{22}, R_{33}, R_{12}$.

\subsection{Identified Parameters}

The proposed multi-experiment identification method iteratively minimizes the gap between the simulated and measured data starting from an initial guess for the unknown parameters. The case at hand is chosen since it can be well identified using more classical methods as discussed in Section 2.8. Consequently, the proposed method should return parameters that are similar to the classical parameter set. Table 1 shows the two sets of parameters. Most parameters are nearly identical between the two sets; most notably the elastic parameters and the anisotropy parameters. The plastic parameters $\sigma_{0}, h$ and $m$ have changed significantly. However, even with seemingly large changes in these parameters, the stress-strain curves obtained from both parameter sets are remarkably similar (see Figure 5). 
Table 1: Identified parameters using the classical method and the discussed multi-experiment framework

\begin{tabular}{l|cc|ccc|ccc}
\hline & $E$ & $\nu$ & $\sigma_{0}$ & $h$ & $m$ & $R_{22}$ & $R_{33}$ & $R_{12}$ \\
& $\mathrm{GPa}$ & - & $\mathrm{MPa}$ & $\mathrm{MPa}$ & - & - & - & - \\
\hline Classical & 71.7 & 0.3 & 356 & 602 & 0.5 & 1.04 & 0.89 & 1.07 \\
Multi-Exp. & 70.6 & 0.31 & 330 & 446 & 0.38 & 1.03 & 0.9 & 1.07 \\
\hline
\end{tabular}

To test the dependence on the initial guess, the multi-experiment identification routine was initialized with the "Classical" set and the same set multiplied initial guess were within $10^{-4}$ of the parameters shown in Table 1.

\subsection{Residuals}

The gap between the simulated and measured observables is an integral part of the discussed identification method. The classical identification methods typically depend on indirect residuals, such as the gap between the modeled and measured stress-strain curves. However, the parameters obtained with any identification method can be used to simulate the experiment to construct the full-field residuals as available in full-field identification methods.

The full-field residuals are part of the cost functions discussed in Section 2.3 and are the difference between the simulated and measured quantities

$$
\begin{aligned}
R_{I k t e} & =f_{k e}-\tilde{g}_{k t e}, & & \hat{R}_{I}=\operatorname{rms}\left(R_{I}\right), \\
R_{F t e} & =F_{t e}^{\mathrm{EXP}}-F_{t e}, & & \hat{R}_{F}=\operatorname{rms}\left(R_{F}\right), \\
\vec{R}_{U k t e} & =\sum_{i}^{N_{a}} \vec{\varphi}_{i k e}\left(a_{i t e}^{\mathrm{DIC}}-a_{i t e}\right), & & \hat{R}_{U}=\operatorname{rms}\left(\left|\vec{R}_{U}\right|\right) .
\end{aligned}
$$

In this paper three residuals are discussed, where the image and force residuals ${ }_{415}$ (i.e., $R_{I}$ and $R_{F}$ ) are part of the identification algorithm. The third (displacement) residual $R_{U}$ is optional and available due to the application of global DIC with the same mesh as is used for the identification. Note that the indices $k, t$, and $e$ indicate pixels, time steps and experiments respectively. The number of 
pixels $N_{k e}$ and time steps $N_{t e}$ depend on the experiment, the number of experi-

420 ments $N_{e}$ is three. Consequently $R_{I}$ and $\vec{R}_{U}$ are 3D fields (space and time) per experiment, and the former and latter are respectively scalar and vector fields. The force residual $R_{F}$ is $1 \mathrm{D}$ in time per experiment.

The cost functions (Equations (21), (23) and (29)) are defined as extensive quantities so that they can be aggregated by summation. Consequently, their expectation is equal to the number of observables in the cost function. To facilitate the discussion on the quality of the identification intensive versions of three cost functions are defined here as

$$
\begin{aligned}
\hat{\eta}_{I}^{2} & =\frac{\sum_{e}^{N_{e}} \eta_{I e}^{2}}{\sum_{e} N_{t e} N_{k e}} \\
\hat{\eta}_{F}^{2} & =\frac{\sum_{e}^{N_{e}} \eta_{F e}^{2}}{\sum_{e} N_{t e}} \\
\hat{\eta}^{2} & =\frac{\sum_{e}^{N_{e}} \eta_{e}^{2}}{\sum_{e} N_{t e} N_{k e}+N_{t e}}
\end{aligned}
$$

The first two are not used for identification purposes, but they are useful as indicators of the agreement of the individual data sets with the parameter set 425 obtained with the identification set. All three have expectations of unity under the condition that the assumptions of measurement noise and model error are valid.

Table 2 shows the displacement $\bar{R}_{U}$, image $\bar{R}_{I}$ and force $\bar{R}_{F}$ residuals for the two parameter sets given in Table 1 as well as the image residual obtained using non-integrated DIC. The displacement and image residuals are only marginally larger than their respective uncertainties for both the "Classical" and the "MultiExp." parameter sets. However, the force residual is considerably larger than its expected uncertainty for both identification cases. The same is expressed in the intensive cost function values $\hat{\eta}$, which are expected to be close to unity for the image part of the cost function while being far above unity for the force contribution. However, both identification methods return similar residuals with the multi-experiment identification method residuals being lower for all quantities. 
Table 2: Residuals remaining after convergence for both identification cases, $\hat{\eta}$ are the intensive cost function residuals (Equation (51))

\begin{tabular}{l|ccc|ccc}
\hline & $\hat{R}_{U}[\mathrm{px}]$ & $\hat{R}_{I}[\%]$ & $\hat{R}_{F}[\mathrm{~N}]$ & $\hat{\eta}_{I}$ & $\hat{\eta}_{F}$ & $\hat{\eta}$ \\
\hline Classical & 0.24 & 3.13 & 380 & 0.76 & 76 & 0.76 \\
Multi-Exp. & 0.22 & 2.78 & 336 & 0.67 & 67 & 0.68 \\
DIC & - & 1.80 & - & - & - & - \\
\hline
\end{tabular}

An average gap between the simulated and measured forces of $336 \mathrm{~N}$ compared to a peak force of $10 \mathrm{kN}$ may be considered both reasonable or unrea${ }_{440}$ sonable depending on the understanding of the experimental setup. The fact is that there are significant forces present in the measured data that are not accounted for by the model. These differences can have a number of sources, not limited to constitutive model error, geometrical model error, synchronization errors and measurement artifacts. The model error is present in both identification methods. However, the advantage of the proposed method is that the model error is now clearly visualized to allow the user of the method to judge whether this error is tolerable or not. Additionally, the signature of the model error can be analyzed to propose model enrichments that can account for the missing kinematics [20].

450 The $\hat{\eta}$ values reported in Table 2 are unexpectedly smaller than unity. First let us note that the image intensive cost function $\hat{\eta}_{I}$ is dominant in the global cost function, essentially because of the very large number of pixels as compared to the number of force measurements. Even, if $\hat{\eta_{F}}$ is very large as a result of a model error, this does not increase much the (intensive) global residual. The most likely explanation for $\hat{\eta}_{I}$ being smaller than 1 is that the estimate for the image uncertainty $\gamma_{I}$ was high. The 20 images that were used to estimate image uncertainty may have been contaminated by a small but non-zero load. A deeper analysis of the uncertainty estimate may resolve this. However, it is considered out of the scope of this paper. A possible consequence is that the current identification method slightly overemphasized the force data.

Figure 12 shows the residuals for the parameter set obtained using the clas- 
sical identification method. It presents the residuals as three columns, one per experiment, with seven sub-figures each. The first two rows are the displacement residual between the simulated displacements and those measured with non-integrated DIC (see Equation (48)) for one time and one space cross-section. The third row reports the image residual (Equation (50)) and the fourth and final row shows the residual force (Equation (49)) as a function of time.
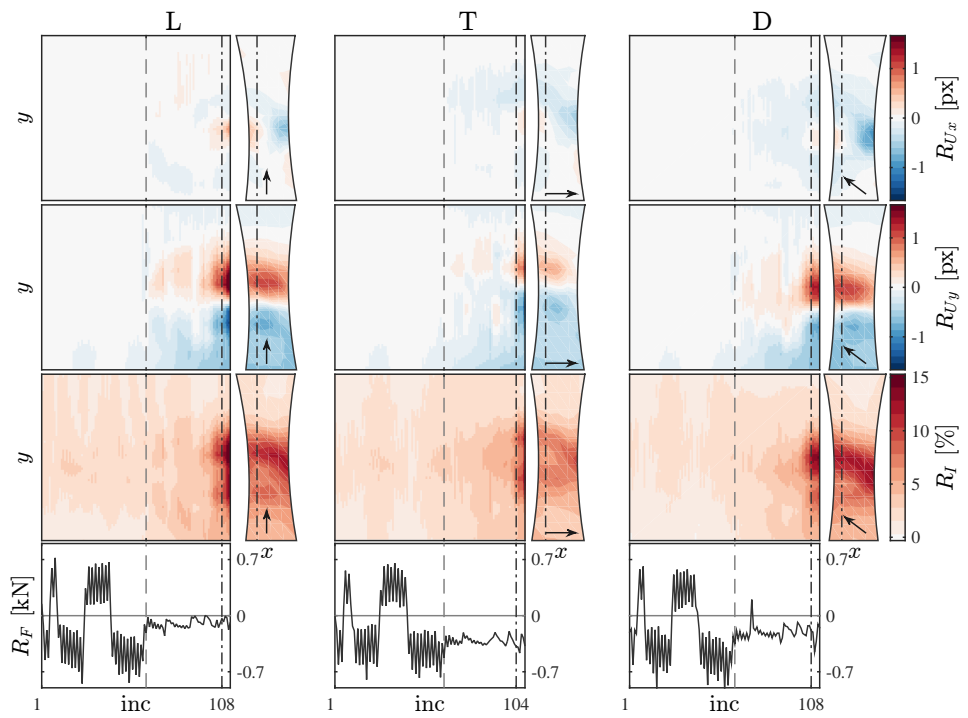

Figure 12: Full-field residuals in terms of displacement (first two rows), gray levels ( $3^{\text {rd }}$ row) and force ( $4^{\text {th }}$ row) for all three experiments when using the "Classical" parameter set as shown in Table 1.

The vertical dashed lines in the figure indicate the transition from the elastic part of the experiment to the plastic part roughly in the center of the time axis (see also Figure 3). The displacement and image residuals show a relatively low residual before this instant of time, and then gradually increase as plasticity is mobilized in the sample. This behavior is similar for all three experiments. However, the residuals for the T-sample are generally lower. For the force residual $\left(4^{\text {th }}\right.$ row) the inverse is shown, with high residuals in the elastic part and lower residuals after the onset of plasticity. Additionally, the force residual is lowest for the L-sample, which is expected because the elastoplastic parameters 
(i.e., $E, \nu, \sigma_{0}, h$ and $m$ ) were all obtained from the L-experiment data only. The high residuals in force in the elastic part correlate with the load-unloading cycles in the elastic regime and clearly indicate a phenomenon present in the experiment that is not modeled. This type of model error is common in identification, and the consequence of using real data instead of synthetic data. No further investigation of the source of this model error will be carried herein as it lies outside of the scope of the paper.

Similar to Figure 12, Figure 13 shows the full-field residuals, now for the parameter set calibrated with the discussed identification method. As was already known from the global residuals (Table 2), the multi-experiment residuals are generally lower for the multi-experiment identification as compared to the classical method. This is expected, since the multi-experiment framework is targeting these residuals directly in its cost function. Additionally, the residuals are more homogeneously distributed among the three experiments.
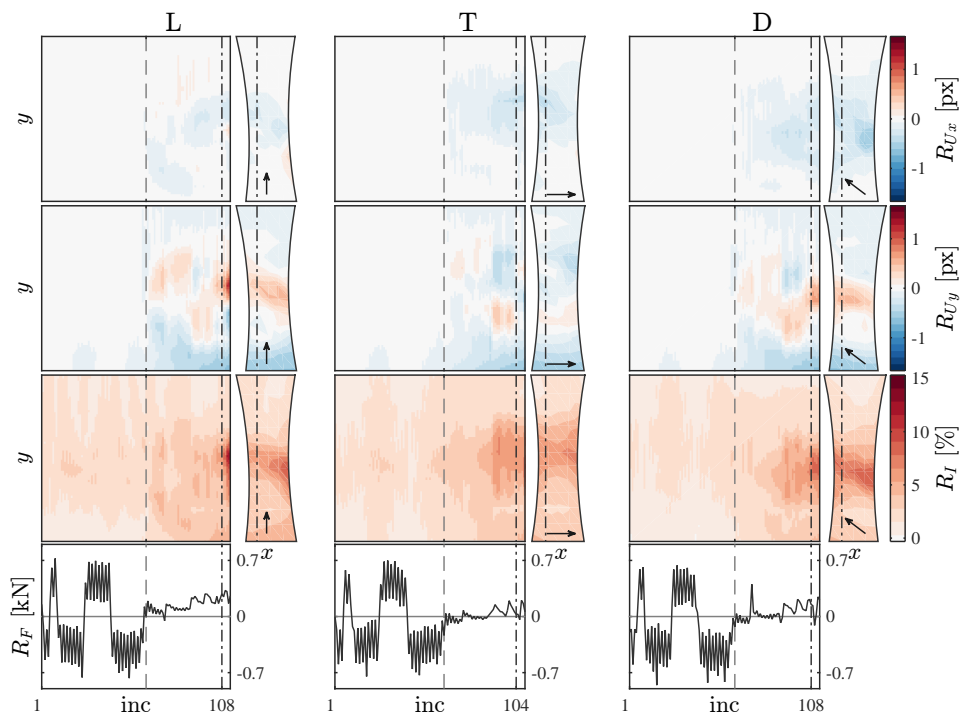

Figure 13: Full-field residuals in terms of displacement (first two rows), gray levels ( $3^{\text {rd }}$ row) and force $\left(4^{\text {th }}\right.$ row $)$ for all three experiments when using the multi-experiment parameter set as shown in Table 1. 


\section{Conclusions}

An identification method is proposed that aggregates the experimental data from multiple sources of multiple experiments into an identification method to calibrate a single set of constitutive parameters. The method is based on Bayesian principles, thereby automatically accounting for the uncertainty of each data source to find their optimal weighting. An extractor is constructed to identify the set of unknown parameters considering all data that can reliably be represented by the chosen numerical model. The method provides an identification framework that naturally encompasses and extends on previously published global DIC and Integrated-DIC frameworks. However, the proposed method is not limited to full-field data and works equally well with other measurement types, such as, load-cells, strain gages.

As a proof of concept, the method is applied to the identification of an 8 parameter isotropic elastoplastic model with a planar anisotropic yield surface.

${ }_{505}$ The parameters calibrated with the proposed method are very similar to those using more classical methods. Moreover, the residual gap between the measured and modeled quantities is significantly reduced using the proposed method. The test case is chosen to illustrate this comparison, but it is emphasized that the proposed method is applicable to a much wider range of constitutive models and inhomogeneous experiments.

One of the challenges in the identification of complex material models is dealing with non-uniqueness. Often there are multiple solutions that minimize the identification cost function. For cases that are not interested in the parameter values directly, picking any of the parameter sets will suffice, provided the application of the calibrated model remains close to the identification regime. However, often the purpose of identification is to study the constitutive model directly. In those cases the parameter values are important. A way to discriminate between the solution sets is to enrich the identification with an additional experiment, designed specifically to trigger mechanisms previously not or not sufficiently present. Within this paper methods are discussed to aid in de- 
signing these experiments by means of analyzing the sensitivity of promising experiments.

The proposed method is based on the concept of transporting uncertainty along the experimental chain up to parameter identification. Consequently, it provides uncertainty assessments of the calibrated parameters. It is based on a Gaussian model of the acquisition noise. Although a reasonable assumption for acquisition noise, such an assessment does not include any non-stochastic effects. The major critical point not yet addressed is the impact of model error. Most, if not all, parameter identification methods leave a residual gap between the measured and modeled quantities. Often, the amplitude of this gap is more significant than the uncertainty due to random noise. Comparing the residual gap of the proposed method to more classical and trusted identification methods reveals that this issue is only mildly improved with the proposed method. The key difference is that the proposed method shows the residual gap very visually and thus provides means of assessing whether the gap is acceptable. For the example discussed herein, the most likely cause of the residual gap is a limited model (be it from the constitutive law, experimental control, or measurement acquisition) that is not capable of describing the experiment correctly at the transition from loading to unloading and for higher plastic strains. One way 540 to address this issue is the successive enrichment of the model with more parameters [20]. One should take care of the risk of degeneracies. However, the proposed method provides various tools for progressing along this challenging path.

\section{Acknowledgement}

545 It is a pleasure to acknowledge the support of BPI France within the "DICCIT" project. 


\section{References}

[1] O. Lademo, O. S. Hopperstad, M. Langseth, An evaluation of yield criteria and flow rules for aluminium alloys, International Journal of Plasticity 15 (0749) (1999) 191-208.

[2] D. Banabic, F. Barlat, O. Cazacu, T. Kuwabara, Advances in anisotropy and formability, International Journal of Material Forming 3 (3) (2010) 165-189. doi:10.1007/s12289-010-0992-9.

[3] M. A. Sutton, J.-J. Orteu, H. Schreier, Image Correlation for Shape, Motion and Deformation Measurements, Springer US, Boston, MA, 2009.

[4] M. A. Sutton, Computer Vision-Based, Noncontacting Deformation Measurements in Mechanics: A Generational Transformation, Applied Mechanics Reviews 65 (5) (2013) 1-23. doi:10.1115/1.4024984.

URL http://appliedmechanicsreviews.asmedigitalcollection.

[5] M. Grediac, F. Sur, 50th anniversary article: Effect of sensor noise on the resolution and spatial resolution of displacement and strain maps estimated with the grid method, Strain 50 (1). doi:10.1111/str.12070.

[6] S. Avril, M. Bonnet, A.-S. Bretelle, M. Grédiac, F. Hild, P. Ienny, F. Latourte, D. Lemosse, S. Pagano, E. Pagnacco, F. Pierron, Overview of Identification Methods of Mechanical Parameters Based on Full-field Measurements, Experimental Mechanics 48 (4) (2008) 381-402.

[7] M. Grédiac, F. Hild (Eds.), Full-Field Measurements and Identification in Solid Mechanics, ISTE / Wiley, London (UK), 2012.

${ }_{570}^{578}$ K. T. Kavanagh, R. W. Clough, Finite element applications in the characterization of elastic solids, International Journal of Solids and Structures 7 (1971) 11-23. 
[9] M. H. H. Meuwissen, C. W. J. Oomens, F. P. T. Baaijens, R. Petterson, J. D. Janssen, Determination of the elasto-plastic properties of aluminium using a mixed numerical-experimental method, Journal of Materials Processing Technology 75 (1-3) (1998) 204-211.

[10] O. Ghouati, J. Gelin, Identification of material parameters directly from metal forming processes, Journal of Materials Processing Technology 80-81 (1998) 560-564. doi:10.1016/S0924-0136 (98)00159-9.

[11] T. Pottier, F. Toussaint, P. Vacher, Contribution of heterogeneous strain field measurements and boundary conditions modelling in inverse identification of material parameters, European Journal of Mechanics, A/Solids 30 (3) (2011) 373-382. doi:10.1016/j .euromechsol.2010.10.001. URL http://dx.doi.org/10.1016/j.euromechsol.2010.10.001 features and finite element model updating from full-field strain data, International Journal of Solids and Structures 48 (11-12) (2011) 1644-1657. doi:10.1016/j.ijsolstr.2011.02.010.

URL http://linkinghub.elsevier.com/retrieve/pii/ S0020768311000692

[13] A. Güner, C. Soyarslan, A. Brosius, A. E. Tekkaya, Characterization of anisotropy of sheet metals employing inhomogeneous strain fields for Yld2000-2D yield function, International Journal of Solids and Structures 49 (25) (2012) 3517-3527. doi:10.1016/j.ijsolstr.2012.05.001.

[14] S. Roux, F. Hild, Stress intensity factor measurements from digital image correlation: Post-processing and integrated approaches, International Journal of Fracture 140 (1-4) (2006) 141-157. doi:10.1007/ s10704-006-6631-2.

[15] H. Leclerc, J.-N. Périé, S. Roux, F. Hild, Integrated digital image correlation for the identification of mechanical properties, in: A. Gagalowicz, 
W. Philips (Eds.), Mirage2009, Springer, Berlin Heidelberg, 2009, pp. 161171.

[16] J. Réthoré, A fully integrated noise robust strategy for the identification of constitutive laws from digital images, International Journal for Numerical Methods in Engineering 84 (2010) 631-660.

[17] J. Réthoré, Muhibullah, T. Elguedj, M. Coret, P. Chaudet, A. Combescure, Robust identification of elasto-plastic constitutive law parameters from digital images using 3D kinematics, International Journal of Solids and Structures 50 (1) (2013) 73-85.

[18] F. Mathieu, H. Leclerc, F. Hild, S. Roux, Estimation of Elastoplastic Parameters via Weighted FEMU and Integrated-DIC, Experimental Mechanicsdoi:10.1007/s11340-014-9888-9.

URL http://link.springer.com/10.1007/s11340-014-9888-9

[19] J. Neggers, J. P. M. Hoefnagels, M. G. D. Geers, F. Hild, S. Roux, TimeResolved Integrated Digital Image Correlation, International Journal for Numerical Methods in Engineering 103 (3) (2015) 157-182. doi:10.1002/ nme. 4882 .

[20] J. Neggers, F. Mathieu, F. Hild, S. Roux, N. Swiergiel, Improving full-field identification using progressive model enrichments, International Journal of Solids and Structures 118-119 (2017) 213-223. doi:10.1016/j.ijsolstr. 2017.03 .013$.

URL http://dx.doi.org/10.1016/j.ijsolstr.2017.03.013

[21] A. P. Ruybalid, J. P. M. Hoefnagels, O. van der Sluis, M. G. D. Geers, Comparison of the identification performance of conventional FEM updating and integrated DIC Andre, International Journal for Numerical Methods in Engineering 106 (2016) 298-320.

[22] D. Claire, F. Hild, S. Roux, A finite element formulation to identify damage 
fields: the equilibrium gap method, International Journal for Numerical Methods in Engineering 61 (2) (2004) 189-208.

[29] F. Hild, S. Roux, Comparison of Local and Global Approaches to Digital Image Correlation, Experimental Mechanics 52 (9) (2012) 1503-1519.

[30] S. Cooreman, D. Lecompte, H. Sol, J. Vantomme, D. Debruyne, Elastoplastic material parameter identification by inverse methods: Calculation

[23] M. Grédiac, F. Pierron, Applying the Virtual Fields Method to the identification of elasto-plastic constitutive parameters, International Journal of Plasticity 22 (4) (2006) 602-627. doi:10.1016/j.ijplas.2005.04.007.

URL http://linkinghub.elsevier.com/retrieve/pii/ S0749641905000896

[24] S. Avril, F. Pierron, M. A. Sutton, J. Yan, Identification of elasto-viscoplastic parameters and characterization of Lüders behavior using digital image correlation and the virtual fields method, Mechanics of Materials 40 (9) (2008) 729-742. doi:10.1016/j.mechmat.2008.03.007.

[25] H. Kleemola, M. Nieminen, On the strain-hardening parameters of metals, Metallurgical Transactions 5 (1974) 1863-1866.

URL http://link.springer.com/article/10.1007/BF02644152

[26] R. Hill, A theory of the yielding and plastic flow of anisotropic metals, Proc. Roy. Soc. London 193 (1948) 281-297.

[27] J. Neggers, O. Allix, F. Hild, S. Roux, Big Data in Experimental Mechanics and Model Order Reduction: Today's Challenges and Tomorrow's Opportunities, Archives of Computational Methods in Engineeringdoi:10.1007/s11831-017-9234-3.

[28] G. Besnard, F. Hild, S. Roux, "Finite-Element" Displacement Fields Analysis from Digital Images: Application to Portevin-Le Châtelier Bands, Experimental Mechanics 46 (6) (2006) 789-803.

of the sensitivity matrix, Int. J. Solids Struct. 44 (13) (2007) 4329-4341. 
[31] C. Henninger, S. Roux, F. Hild, Enriched kinematic fields of cracked structures, International Journal of Solids and Structures 47 (24) (2010) 3305-3316. doi:DOI:10.1016/j.ijsolstr.2010.08.012.

URL http://www.sciencedirect.com/science/article/ B6VJS-50SXC5X-1/2/b2f 4345fb7d3b32d3222b2235768a2fe

[32] F. Mathieu, F. Hild, S. Roux, Identification of a crack propagation law by digital image correlation, International Journal of Fatigue 36 (1) (2012) 146-154. doi:10.1016/j.ijfatigue.2011.08.004.

URL http://dx.doi.org/10.1016/j.ijfatigue.2011.08.004

[33] A. Baldi, Residual Stress Measurement Using Hole Drilling and Integrated Digital Image Correlation Techniques, Experimental Mechanics 54 (3) (2013) 379-391. doi:10.1007/s11340-013-9814-6.

URL http://link.springer.com/10.1007/s11340-013-9814-6

[34] M. B. R. Bertin, F. Hild, S. Roux, Optimization of a cruciform specimen geometry for the identification of constitutive parameters based upon full-field measurements, Strain 52 (4) (2016) 307-323. doi:10.1111/str.12178.

[35] D. Lindner, F. Mathieu, F. Hild, O. Allix, C. Ha Minh, O. Paulien-Camy, On the evaluation of stress triaxiality fields in a notched titanium alloy sample via integrated $\{$ DIC\}, J. Appl. Mech. 82 (7) (2015) 71014. doi: $10.1115 / 1.4030457$.

[36] A. N. Tikhonov, V. Y. Arsenin, Solutions of ill-posed problems, J. Wiley, New York (USA), 1977.

[37] ASTM, E8 / E8M-15a, Standard Test Methods for Tension Testing of Metallic Materials, Vol. 03.01, ASTM International, West Conshohocken, PA (USA), 2015.

[38] ASTM, E517-00(2010), Standard Test Method for Plastic Strain Ratio R for Sheet Metal, ASTM International, West Conshohocken, PA (USA), 2010. doi:10.1520/E0517-00R10. 
[39] J. Neggers, F. Mathieu, F. Hild, S. Roux, N. Swiergiel, Improving full-field identification using progressive model enrichments, International Journal of Solids and Structures early acce. doi:10.1016/j.ijsolstr.2017.03.013.

[40] Dassault Systèmes Simulia, Abaqus Analysis User's Manual, Vol. 3 Material, Dassault Systèmes Simulia, 2012.

[41] H. Leclerc, J. Neggers, F. Mathieu, F. Hild, S. Roux, Correli 3.0, Iddn.fr.001.520008.000.s.p.2015.000.31500, Agence pour la Protection des Programmes, Paris (France) (2015).

[42] F. Hild, A. Bouterf, L. Chamoin, H. Leclerc, F. Mathieu, J. Neggers, F. Pled, Z. Tomičević, S. Roux, Toward 4D mechanical correlation, Advanced Modeling and Simulation in Engineering Sciences 3 (1) (2016) 17. doi:10.1186/s40323-016-0070-z.

URL http://amses-journal.springeropen.com/articles/10.1186/ s40323-016-0070-z

[43] A. Tarantola, Inverse Problem Theory and Methods for Model Parameter Estimation, 2005.

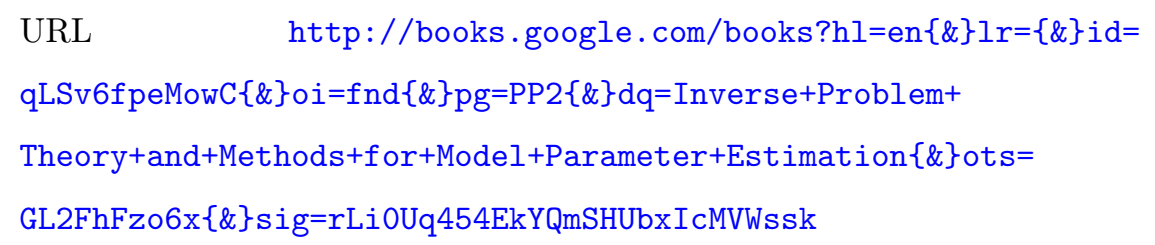

